\title{
Winged scapula caused by rhomboid paralysis: a case report
}

\author{
MARIA GRAZIA BENEDETTI ${ }^{1}$, ALESSANDRO ZATI ${ }^{1}$, SILVIA BONFIGLIOLI STAGNI ${ }^{1}$, ISABELLA \\ FUSARO $^{1}$, ROBERTA MONESI ${ }^{1}$, ROBERTO ROTINI ${ }^{2}$
}

${ }^{1}$ Physical Medicine and Rehabilitation Unit, Istituto Ortopedico Rizzoli, Bologna Italy

${ }^{2}$ Shoulder and Elbow Surgery Unit, Istituto Ortopedico Rizzoli, Bologna, Italy

\begin{abstract}
Scapular winging secondary to dorsal scapular nerve (DSN) damage is an underestimated condition. It is often caused by entrapment of the nerve due to a hypertrophic middle scalene muscle, or by stretching of the DSN during traumatic movements. The condition has also been attributed to myofascial pain syndrome of the rhomboids with entrapment of the DSN. The non-specific symptomatology reported by patients is often incorrectly diagnosed, and this can result in a high level of disability of the upper limb.

A clinical case of misdiagnosed dorsal scapula entrapment is presented. Satisfactory shoulder function recovery, pain relief and reduction of disability were obtained after correct diagnosis of the condition and a comprehensive rehabilitation approach.
\end{abstract}

Keywords: winged scapula, scapular dorsal nerve paralysis, rehabilitation.

\section{Introduction}

The most frequent cause of unilateral scapular winging is damage to the spinal accessory and long thoracic nerves that, respectively, innervate the trapezius and serratus anterior muscles; rarely, the condition is caused by a lesion of the dorsal scapular nerve (DSN) that innervates the rhomboid muscle and sometimes the levator scapulae (1).

\section{Corresponding Author:}

Maria Grazia Benedetti, MD

Physical Medicine and Rehabilitation Unit

Istituto Ortopedico Rizzoli

Via Pupilli 1, 40124 Bologna, Italy

E-mail: benedetti@ior.it
DSN injuries can give rise to a well-defined chronic pain syndrome, often referred to as DSN syndrome $(1,2)$. Patients usually complain of shoulder pain along the medial border of the scapula, occasionally radiating to the lateral surface of the arm and forearm, winging of the scapula on elevating the arm above the head, dysfunction and impairment.

Weakness and hypotrophy of the rhomboid muscle or levator scapulae muscle, or both, can also be present (1). Although DSN entrapment used to be considered a rare condition, more recently Sultan and Younis ElTantawi (1) demonstrated that it is more common than previously estimated. DSN entrapment can be a frequent underlying causative factor for dorsalgia and routine assessment of the DSN in cases of dorsalgia is recommended. We describe the case of a patient in whom a DSN lesion was not correctly diagnosed as it was clinically masked by a depressive condition following a major trauma, and by disuse pathology of the upper limb due to shoulder pain.

\section{Case description}

A 24-year-old woman came to our attention four years after a crash in which she had suffered a fracture of the pelvis and abdominal and chest trauma. For one year she had been experiencing dorsalgia and continuous severe left shoulder pain irradiating to the left upper limb, with marked functional impotence. On hospital admission the patient showed left scapular winging and an elevated left shoulder with painful tonic contracture of the trapezius (this also showed increased bulk), scalene and pectoral muscles. As a result of these conditions, the shoulder was fixed in anteposition with internal rotation, and shoulder elevation and arm abduction were impeded (Fig. 1A). The passive range of motion (ROM) of the left shoulder consisted of $0-50^{\circ}$ of fle- 
xion, and $0-40^{\circ}$ of abduction; external rotation and extension were not possible; internal rotation of the hand at the trochanter. The left arm was held at the side and no active motion was possible. Tingling and numbness in the left upper limb were also present, associated with severe weakness of the upper limb muscles with tremor on initiating active movements. The patient's Constant-Murley score on admission was 8 points. She presented with depressive symptoms which she had developed following her accident and the resulting severe trauma; she was worried about her arm, and in pain.

Shoulder X-ray revealed scapular winging without evident bone fractures. Ultrasound imaging demonstrated no lesions of the rotator cuff; the trapezius and supraspinatus muscles appeared slightly thickened, consistent with contracture. MRI of the cervical spine did not reveal any C5 radiculopathy, while an EMG study showed signs of denervation of the left rhomboid muscle (probable lesion of the DSN). A 3D computed tomography (CT) scan of the shoulder affected by scapular winging was performed in order to obtain a complete view of the skeletal structure of the joint (Fig. 2). The examination confirmed the presence of a winged scapula with preserved muscle trophism. Following a psychiatric consultation, treatment based on agomelatine, associated with the support of a psychologist, was suggested in order to combat the post-trauma depression syndrome.

The patient underwent an initial cycle of rehabilitation consisting of pharmacological pain control and physi- cal therapy which comprised ultrasound treatment of the left shoulder, continuous passive motion, back and shoulder massage, lymphatic drainage of the upper limb, transcutaneous electrical nerve stimulation applied to the left shoulder and arm, shoulder kinesitherapy, EMG biofeedback (to release the trapezius and better activate the rhomboid), and electrical stimulation of the deltoid. In order to further improve the shoulder ROM, a 100 IU dose of botulinum toxin A (BTA) was injected into the left trapezius, in two sites, to relax the trapezius.

A second rehabilitation treatment period was planned after 1 month. Contracture of the left trapezius was significantly reduced even if the winging scapula and the left shoulder elevation were still present (Fig. 1B). The pain gradually improved and the patient gained muscle strength and an active ROM at the left shoulder: $80^{\circ}$ of flexion, $60^{\circ}$ of abduction, $45^{\circ}$ of external rotation, internal rotation to the buttock, $15^{\circ}$ of extension. After a further six months, since the patient still showed a marked hyperactivation of the pectoralis major, this muscle was treated with $100 \mathrm{IU}$ of BTA, injected in two sites.

At the end of the last cycle of rehabilitation, 14 months after the initial admission, the patient showed a further reduction of her symptoms, displaying a satisfactory recovery of active ROM (active flexion: $160^{\circ}$, abduction: $90^{\circ}$, external rotation: $45^{\circ}$, internal rotation of the hand to L3, extension: $20^{\circ}$ ), as well as satisfactory muscle strength and functional use of the limb in activities of daily living (Fig. 1C).
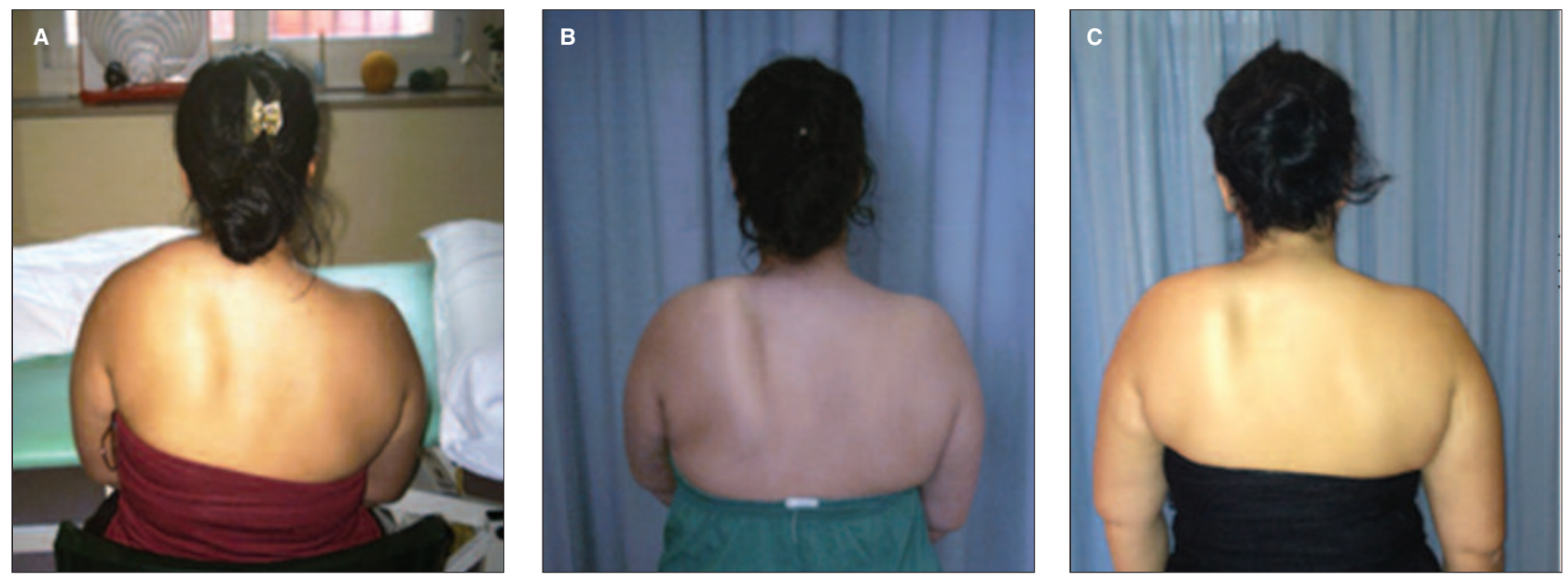

Figure 1. Clinical presentation of the patient on admission to hospital (A), after 2 months of physical therapy (B), and after 14 months (C). 


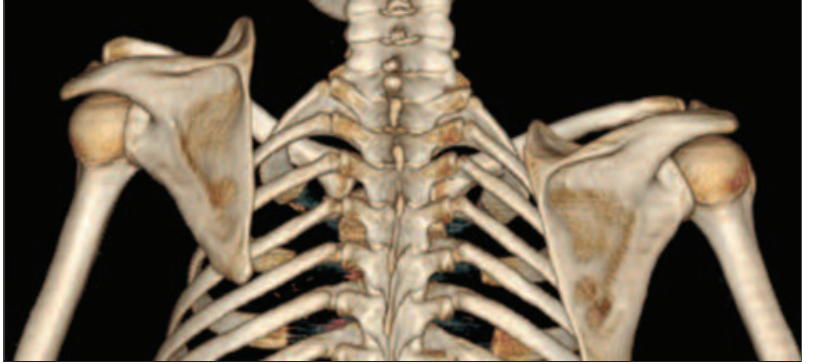

Figure 2. 3D CT reconstruction of the left shoulder.

The patient was discharged from hospital with a Constant-Murley score of 45 points, and continued rehabilitation as an outpatient at a clinic near to her own home.

\section{Discussion}

The different etiological hypotheses that have been advanced to explain DSN syndrome include trauma to the brachial plexus and nerve entrapment $(1,3)$. EMG assessment is considered fundamental for a correct diagnosis (1). In the present case the lesion was probably related to the chest trauma. Since the symptoms were not specific, the diagnosis in the present case was initially missed, with the result that the patient developed global upper limb dysfunction. Contraction of the trapezius muscle and pectoralis major compensated for the dysfunction caused by the rhomboid paralysis and consequent scapular winging. The trapezius can, in fact, substitute for rhomboid muscle in scapular adduction movement. The use of botulinum toxin to treat contracture of the trapezius in scapula dyskinesia has already been reported in the literature $(4,5)$, and shown to allow reduction of winging. In the present case, its use both in the trapezius and in the pectoralis major was found to be effective. According to Sultan and Younis El-Tantawi (1), poor definition of the characteristics and extension of the pain is the cause of frequent misdiagnoses, and the condition can be confused with other shoulder chronic pain syndromes if the DSN is not specifically explored. In this patient, a multidisciplinary approach to the rehabilitation, appropriate diagnostics, and targeted treatment limited the patient's disability and thus resulted in a satisfactory outcome.

\section{References}

1. Sultan HE, Younis El-Tantawi GA. Role of dorsal scapular nerve entrapment in unilateral interscapular pain. Arch Phys Med Rehabil. 2013;94:1118-1125.

2. Meininger AK, Figuerres BF, Goldberg BA. Scapular winging: an update. J Am Acad Orthop Surg. 2011;19:453-462.

3. Akgun K, Aktas I, Terzi Y. Winged scapula caused by a dorsal scapular nerve lesion: a case report. Arch Phys Med Rehabil. 2008;89:2017-2020.

4. Fusaro I, Orsini S, Bellenghi C, Smeraldi S, Rotini R, Sinapi F. Treatment of scapula dyskinesia with botulin toxin: two case reports. Musculoskelet Surg. 2010;94 Suppl 1:S95-98.

5. Cossu G, Melis M, Melis G, Ferrigno P, Molari A. Persistent abnormal shoulder elevation after accessory nerve injury and differential diagnosis with post-traumatic focal shoulder-elevation dystonia: report of a case and literature review. Mov Disord. 2004;19:1109-1111. 